\title{
Response of Critical Tube Diameter Phenomenon to Small Perturbations for Gaseous Detonations
}

Submitted: 25 January 2013 / Revised: 18 June 2013

\begin{abstract}
In this study, an experimental investigation is carried out to further study the critical tube diameter problem for the transmission of gaseous detonation from a confined tube into a sudden open space in both regular mixtures, those highly diluted with argon and irregular mixtures of which the cellular detonation is highly unstable. The two commonly postulated modes of failure consisting of one by a local failure mechanism that is linked to the effect of instabilities for undiluted mixtures, and the other due to the excessive curvature of the global front in mixtures highly diluted with argon, are further investigated through experiments. To discern between these mechanisms in the different mixtures, flow perturbations are imposed by placing a minute obstacle with small blockage ratio at the tube exit diameter just before the detonation diffraction. Results show that the perturbation only has an effect in undiluted mixtures resulting in the decrease of the critical pressure for successful detonation transmission. In other words, the flow fluctuation caused by the small obstacle produces transmission and this result seems to indicate that local hydrodynamic instabilities are significant for the detonation diffraction in undiluted unstable mixtures. On the other hand, the results appear to be the same for both unperturbed and perturbed cases in highly argon diluted mixtures. The small blockage only produces flow fluctuations and does not substantially influence the global curvature of the
\end{abstract}

N. Mehrjoo, Rocco Portaro, H.D. Ng

Mechanical and Industrial Engineering, Concordia University

Montréal, Québec, H3G 1M8, Canada

Tel.: (514) 848-2424 x 3177

Fax: (514) 848-3175

E-mail: hoing@encs.concordia.ca

J.H.S. Lee

Department of Mechanical Engineering, McGill University

Montréal, Québec, H3A 2K6, Canada

B. Zhang

State Key Laboratory of Explosion Science and Technology, Beijing Institute of Technology, Beijing, 10081, China. (Currently at the School of Resources and Environmental Engineering, ECUST, Shanghai, 200237, China) emergent detonation wave as illustrated in the numerical gasdynamic simulation and hence, it shall not affect the failure mechanism of the stable detonation in highly argon diluted mixtures. The observed phenomenon is also shown to be geometry independence of the obstacle even for the irregular mixtures of which the cellular detonation is highly unstable. This means that as the blockage ratio for a specific tube is kept constant, regardless of its blockage configuration the imposed perturbation shows almost an identical behavior for the wave transmission in irregular mixtures while has no major effect on this detonation dynamic parameter in regular ones.

Keywords Detonation · Critical tube diameter · Failure mechanism - Mixture sensitivity · Flow perturbation

PACS 47.40.Rs

\section{Introduction}

The critical tube diameter, $d_{c}$ refers to the minimum tube diameter above which a self-sustained detonation can transmit into an unconfined space without failure. The critical tube diameter problem has been studied extensively in the past. It is one of the dynamic detonation parameters, that has the most accessible and accurately measured parameter in order to quantify sensitivity and to describe the dynamic behavior of an explosive mixture $[1,2]$. In addition, it also applies to Pulsed Detonation Engine (PDE) research especially for tube initiator geometries, e.g., when a detonation transmits from the small pre-detonator to the main thrust tube of the pulse detonation engine [3].

Although no complete theory of critical tube diameter has yet been developed, experimentally it has been well established $[4,5]$, that for many common hydrocarbon fuels-oxygen or -air mixtures, the critical tube diameter can be scaled universally through the characteristic cell size of the detonation front with $d_{c}=13 \lambda$. Nevertheless, studies have shown that this empirical relationship breaks down for mixtures that are highly diluted with 
argon or for mixtures of which the detonation front is highly regular. The critical conditions can vary as much as $d_{c} \sim 20$ to $30 \lambda$ for mixtures with a significant amount of argon dilution [6-9]. This effect appears to result from the unstable nature or difference in regularity between the detonation fronts in undiluted (unstable) and diluted (stable) mixtures. As shown from a number of recent studies that for undiluted hydrocarbon mixtures, typically with high activation energy in the chemical reaction, the cellular detonation front is unstable embedded with small scale instabilities and its propagation relies on the interactions of transverse waves $[10,11]$. On the other hand, for detonations in combustible mixtures that have been highly diluted with argon, the detonation front is very regular or appears to be piece-wise laminar where cellular instabilities that do not seem to play a prominent role on the propagation of a stable detonation. The ZND structure is valid and a stable, ZND detonation relies on the classical mechanism of shock-induced auto-ignition [11-13]. The link between the regularity of the instability pattern on the detonation front and the critical tube diameter is discussed in Lee [14]. Lee proposed that the significant difference of the critical tube diameter phenomenon in mixtures with regular and irregular cellular structure is due to the different mechanism of detonation failure. By analyzing the open-shutter photographs by Vasil'ev, Lee pointed out that for unstable detonations, successful transmission is invariably found to originate from localized region in the failure wave, which eventually amplified to sustain the detonation propagation front in the open area. Hence, failure is linked to the suppression of instabilities at which localized explosion centers are unable to form in the failure wave when it has penetrated to the charge axis. While for stable detonations failure is predominantly caused by excessive curvature of the entire detonation front whereby the corner expansion waves distribute the curvature over the detonation surface. These two mechanisms are illustrated schematically in Fig. 1 from [15].

The scope of the present study is to perform new critical tube diameter experiments that could unambiguously discriminate between the two postulated modes of failure. Experiments are carried out in three different combustible mixtures, i.e., stoichiometric mixtures of undiluted acetylene-nitrous oxide, acetylene-oxygen as well as acetylene-oxygen with $70 \%$ of argon dilution, that range from highly irregular (unstable) to regular (stable) mixtures. While explicitly analyzing the gaseous detonation front and visualizing the detonation diffraction process may be challenging, an alternative way to study the detonation structure and to illustrate distinctly the different failure mechanism is to perturb the detonation before the divergence and verify how the phenomenon responds. Hence, small flow perturbations are created purposely by a slender obstacle with minimal blockage ratio to ensure that significant large scale disturbances are not created, and also to minimize shock focusing downstream of the

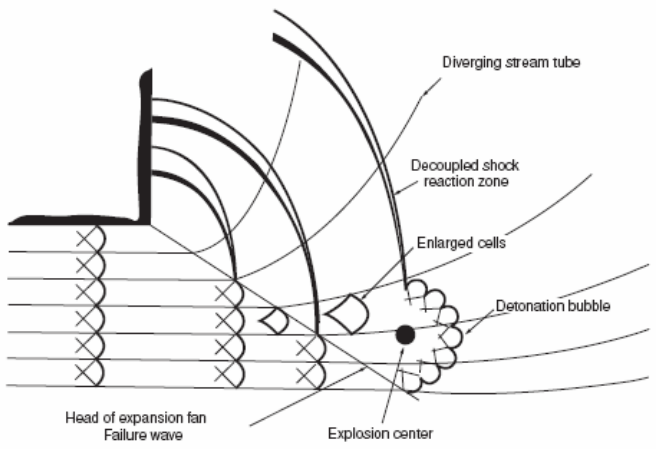

(a)

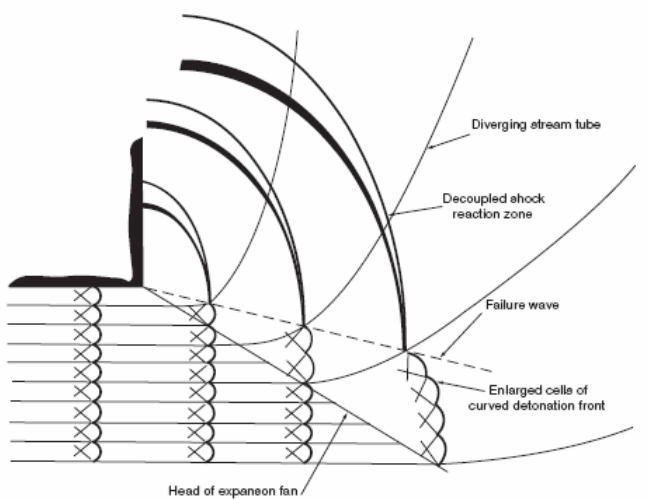

(b)

Fig. 1 A schematic illustrated the two postulated failure mechanisms for a) unstable and b) stable explosive mixtures [15].

obstacle. In addition, several blockage configuration of the obstacle on the wave transmission in mentioned mixtures will also be investigated. The goal is to study the effect of hydrodynamic fluctuations and the significance of these localized instabilities at the detonation fronts in irregular (undiluted) or regular (argon diluted) combustible mixtures, as well as their effect on the successful transmission or failure of the detonation propagation from a confined tube into an abrupt area enlargement.

\section{Experimental Details}

The critical tube diameter experiments were carried out in a modified high-pressure spherical chamber used previously for the measurement of critical energy for direct initiation of spherical detonations [16-18]. The chamber is $20.3 \mathrm{~cm}$ in diameter and $5.1 \mathrm{~cm}$ in wall thickness and it was connected at the top to a $41.8 \mathrm{~cm}$ long vertical circular steel tube of which three different diameter sizes (i.e., $D=15.5 \mathrm{~mm}, 12.7 \mathrm{~mm}$ and 9.13 $\mathrm{mm}$ ) were used for each kind of combustible mixture. The schematic of the critical tube diameter experiment is given in Fig. 2. Stoichiometric mixtures of acetylenenitrous oxide, acetylene-oxygen, and acetylene-oxygen with $70 \%$ argon dilution were tested in this study. The explo- 
sive mixture was prepared beforehand in separate gas bottles by the common method of partial pressure. The gases were allowed to mix in the bottle for at least $24 \mathrm{hr}$ in order to ensure mixture homogeneity. For each experiment, the setup was initially evacuated to approximately $100 \mathrm{~Pa}$ and then filled through the valve with mixtures at various initial test pressures $p_{o}$. The initial pressure measurement was taken via an Omega model PX309030AI pressure transducer (0 - $30 \mathrm{psi})$ with an accuracy of $\pm 0.25 \%$ full scale. A planar Chapman-Jouguet (CJ) detonation was first initiated with a high-voltage spark ignition source, which consisted of a high voltage power supply, capacitor bank, a gap-switch, a trigger module (TM-11A, PerkinElmer Inc.) and a slender coaxial electrode mounted at the top of the vertical steel tube [16]. To ensure that a fully-developed CJ detonation was established in the confined tube, a fiber optic probe located close to the vertical tube exit was also used in some tests to measure the time of the arrival and hence, to deduce the detonation velocity [19]. The self-sustained CJ detonation wave subsequently emerged into a large spherical bomb chamber at the other end of the vertical steel tube. A piezoelectric pin (CA-1136, Dynasen Inc.) was mounted at the bottom wall of the chamber to measure the time-of-arrival of the wave and to determine whether the emergent detonation was successfully transmitted into the open space or not. The measurement of traveling time of waves from ignition to the arrival time to the shock pin is sufficient to determine 'go/no go' due to the time scale difference between arrival times for high-speed deflagration and detonation being very different. For each experimental condition (i.e., mixture composition, initial pressure $p_{o}$ and tube diameter $D$ ), experiment was performed 8 times to ensure repeatability of the results. The sensitivity of the mixtures was varied by the initial pressure. The critical condition for each mixture is characterized by the critical pressure below which the detonation fails to emerge into the large spherical chamber.

To investigate the effect of small perturbation on the critical tube diameter phenomenon, flow disturbance was generated by the insertion of a slender needle at the exit diameter of the vertical tube, see Fig. 2. Three different sizes of needles were inserted for the three different tube diameters in order to keep the blockage ratio constant; i.e., by using the biggest needle of diameter $1.0 \mathrm{~mm}$ for the big size tube $(D=15.5 \mathrm{~mm})$, the needle of diameter $0.8 \mathrm{~mm}$ for the mid-size tube $(D=12.7 \mathrm{~mm})$ and the needle of diameter $0.6 \mathrm{~mm}$ for the small-size tube $(D$ $=9.13 \mathrm{~mm}$ ). The blockage ratio is kept approximately equal to 0.08 .

It is worth noting that for the mixtures chosen in this investigation, dilution with argon changes the stability of the cellular detonation and makes the reaction rate less temperature sensitive or the detonation more stable [12]. Although the dilution effect of argon lowers the energetic of the mixture, on the other hand it also

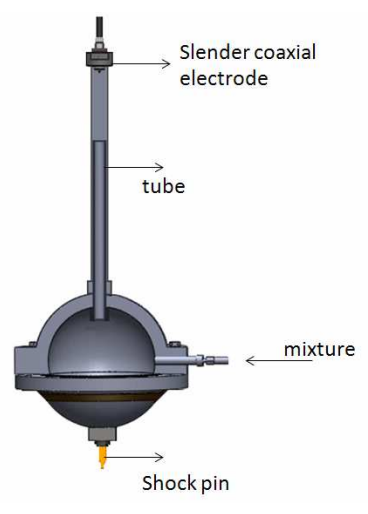

(a)

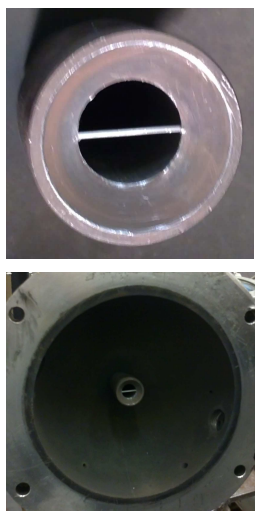

(b)
Fig. 2 Experimental setup: a) experimental apparatus; and b) needle insertion at the tube diameter exit to generate small flow perturbations.

causes an increase in the specific heat ratio, leading to an effect of increasing the shock temperature and hence, there should be no substantial weakening in diluted mixtures at critical conditions for detonation transmission of each mixture and the magnitude of the gasdynamic effect of the obstacle is comparable. In this study, the effect of obstacle is always compared relative to perturbed and unperturbed cases for the same mixture.

\section{Results and Discussion}

In this study, three kinds of gas mixtures were considered, i.e., stoichiometric $\mathrm{C}_{2} \mathrm{H}_{2}-\mathrm{N}_{2} \mathrm{O}, \mathrm{C}_{2} \mathrm{H}_{2}-\mathrm{O}_{2}$, and $\mathrm{C}_{2} \mathrm{H}_{2}-\mathrm{O}_{2}$ diluted with $70 \%$ of argon. These three mixtures qualitatively represent the cases for highly irregular (highly unstable), unstable and stable (highly regular) cellular detonation structures, respectively. Experimental observation on the regularity of the cell size pattern, made it possible to compute the stability parameter for each mixture as a figure of merit which serves to characterize the sensitivity of the mixtures $[11,13,20]$, and it is given by:

$\chi=\varepsilon_{I} \frac{\Delta_{I}}{\Delta_{R}}=\varepsilon_{I} \Delta_{I} \frac{\dot{\sigma}_{\max }}{u_{C J}^{\prime}}$

where $\varepsilon_{I}$ is the effective normalized activation energy in the induction zone, $\Delta_{I}$ the induction length, and $\Delta_{R}$ the reaction length approximated by the inverse of the maximum thermicity $1 / \dot{\sigma}_{\max }$ multiplied by the CJ particle velocity $u_{C J}^{\prime}$ in shock-fixed coordinates with the thermicity given by:

$\dot{\sigma}=\sum_{i=1}^{N_{s}}\left(\frac{W}{W_{i}}-\frac{h_{i}}{C_{p} T}\right) \frac{d Y_{i}}{d t}$

where $W$ denotes the mean molar mass of the mixture, $C_{p}$ the mixture specific heat at constant pressure, and $Y_{i}$, 


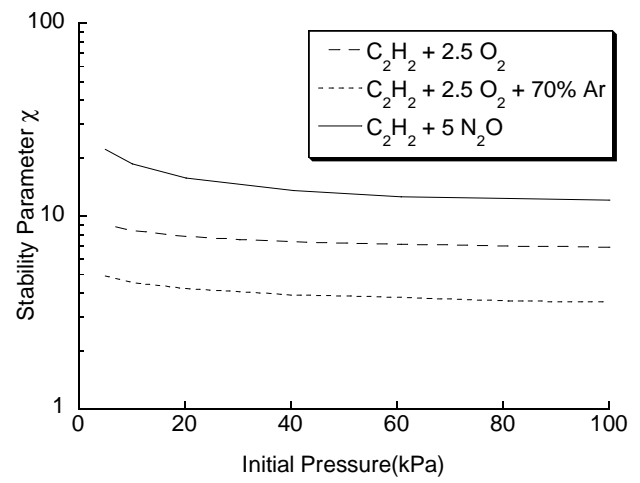

Fig. 3 Stability parameter $\chi$ as a function of the initial pressure $p_{o}$ for stoichiometric $\mathrm{C}_{2} \mathrm{H}_{2}-\mathrm{N}_{2} \mathrm{O}, \mathrm{C}_{2} \mathrm{H}_{2}-\mathrm{O}_{2}$, and $70 \%$ Ar-diluted $\mathrm{C}_{2} \mathrm{H}_{2}-\mathrm{O}_{2}$ combustible mixtures.

$h_{i}$ the mass fraction and the specific enthalpy of specie $i$, respectively. The effective activation energy in the induction process $\varepsilon_{I}$ can be obtained by constant-volume explosion calculations [13]. Chemkin package [21] and the San Diego chemical reaction mechanism [22] were used to compute different chemical kinetics properties including the activation energy and various chemical length scales. The San Diego reaction mechanism has been validated and optimization targets of this mechanism included the detonation of acetylene-oxygen-diluent systems [23]. Figure 3 compares relatively the stability parameter as a function of initial pressure for the three mixtures considered in this study. As the results shown, $\mathrm{C}_{2} \mathrm{H}_{2}-\mathrm{N}_{2} \mathrm{O}$ is the most unstable mixtures among the three with the relatively largest value of $\chi$ and the argon dilution to the $\mathrm{C}_{2} \mathrm{H}_{2}-\mathrm{O}_{2}$ mixture decreases its stability parameter making the mixture more stable. As discussed in [11-13], the variation in this stability parameter can be linked to the regularity of the instability pattern on the detonation front. In other words, both $\mathrm{C}_{2} \mathrm{H}_{2}-\mathrm{N}_{2} \mathrm{O}$ and undiluted $\mathrm{C}_{2} \mathrm{H}_{2}-\mathrm{O}_{2}$ are described by an irregular cellular structure while $70 \%$ Ar-diluted $\mathrm{C}_{2} \mathrm{H}_{2}-\mathrm{O}_{2}$ is characterized by a very regular cellular structure, in accordance to experimental observations of smoke foil measurement $[8,11,20]$. Therefore, echoing the hypothesis by Lee [14] on the critical tube diameter problem, the mechanism of detonation failure should be different in $\mathrm{C}_{2} \mathrm{H}_{2}-\mathrm{N}_{2} \mathrm{O}$ and undiluted $\mathrm{C}_{2} \mathrm{H}_{2}-\mathrm{O}_{2}$ mixtures with irregular cellular detonation structure, to that with diluted $\mathrm{C}_{2} \mathrm{H}_{2}-\mathrm{O}_{2}$ with regular ZND-like detonation structure where instabilities is found to not playing a dominant role.

For each combustible composition, critical tube diameter experiments were performed at different initial pressures to vary the sensitivity of the mixture. Typical traces for a surviving emergent detonation wave from the tube to the open space and a detonation failure case in a stoichiometric $\mathrm{C}_{2} \mathrm{H}_{2}-\mathrm{O}_{2}$ mixture with the tube diameter of $15.5 \mathrm{~mm}$ and initial pressures of $p_{o}=15 \mathrm{kPa}$ and 11 $\mathrm{kPa}$ are illustrated in Fig. 4. It can be seen from Fig. 4a) that at an initial pressure of $15 \mathrm{kPa}$, the arrival time of

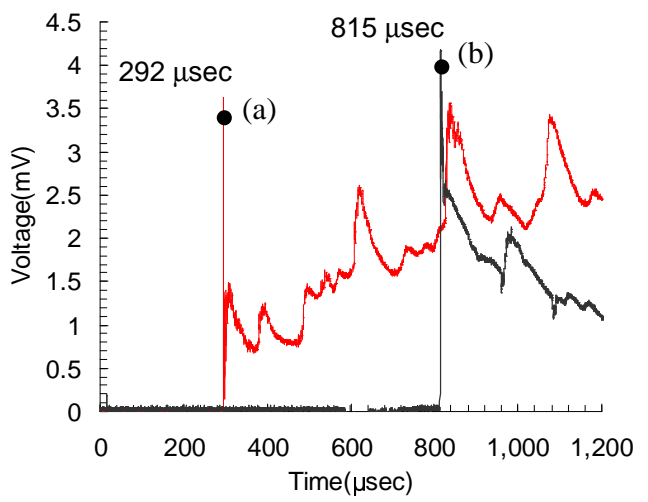

Fig. 4 Time-of-arrival traces for experiments in stoichiometric $\mathrm{C}_{2} \mathrm{H}_{2}-\mathrm{O}_{2}$ mixture (without perturbation) showing: a) successful transmission of the detonation into an unconfined space at $p_{o}=15 \mathrm{kPa}$; and b) unsuccessful transmission at $p_{o}$ $=11 \mathrm{kPa}$.

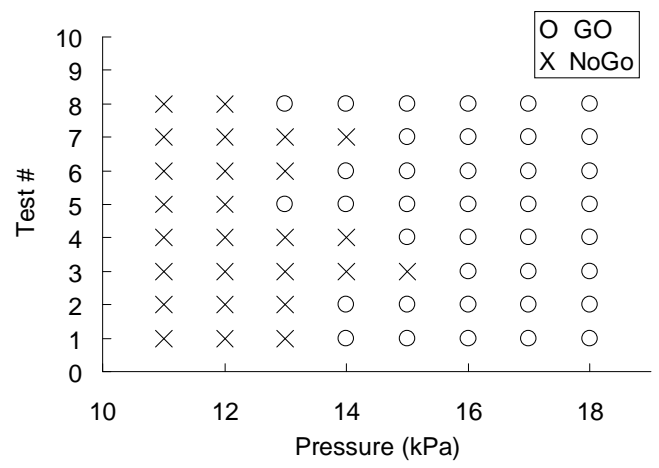

Fig. 5 Test matrix and all go/no go results for the stoichiometric $\mathrm{C}_{2} \mathrm{H}_{2}-\mathrm{O}_{2}$ mixture with the smallest size tube $D=$ $9.13 \mathrm{~mm}$ and with needle perturbation (sample statistics).

the expanding wave is $292 \mu \mathrm{sec}$ when it reaches the shock pin. The velocity of the wave is approximately $2127 \mathrm{~m} / \mathrm{s}$ determined from the ignition to the arrival time to the shock pin and it is about $93 \%$ of the CJ detonation velocity. It shows that at an initial pressure of $15 \mathrm{kPa}$, the tube diameter is above the critical value, thus the planar detonation can successfully transit into a spherical detonation. While for an unsuccessful transmission Fig. 4b) shows when the initial pressure decreases to $11 \mathrm{kPa}$, the expanding wave reaches the piezoelectric pin only at $815 \mu$ sec. Hence the detonation fails after exiting into the free space and the velocity of the expanding wave is roughly $33 \%$ of the CJ velocity value. It is also important to note that the confirmation of a self-sustained CJ detonation in the circular steel tube before diffraction with the range of tube diameters used in the present investigation and the accuracy of the present criterion to distinguish go/no go critical condition for the critical tube diameter problem were discussed and validated in [19].

To ensure statistical convergence and reproducibility of the results, each experiment was repeated 8 times in order to identify accurately the critical pressure value 


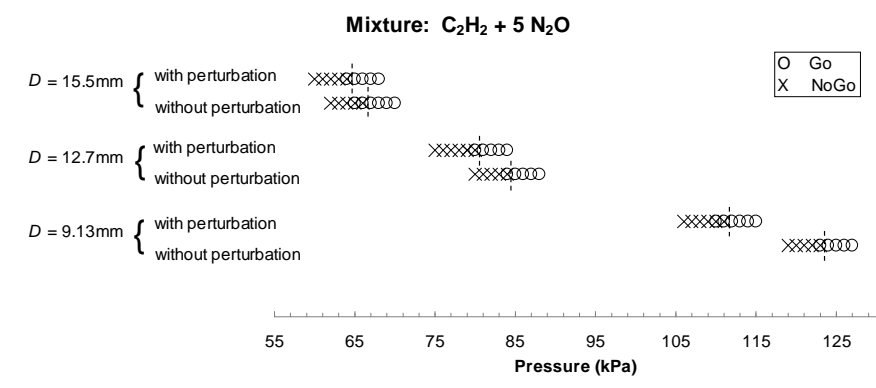

(a)

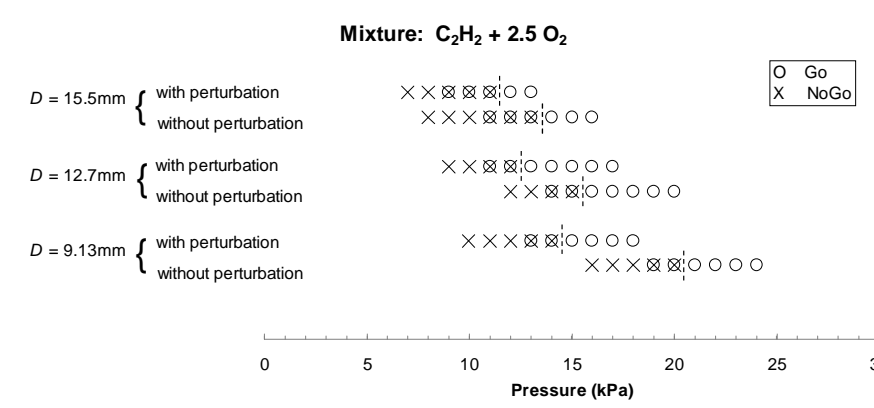

(b)

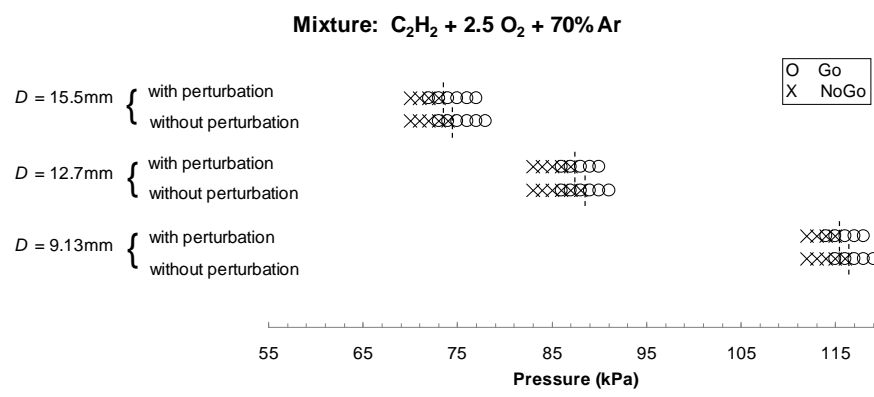

(c)

Fig. 6 Summary of go/no go results for all three combustible mixtures with/without the presence of the needle to create perturbation.

above which can form a spherical detonation at each tube diameter. Figure 5 shows a sample result for the stoichiometric $\mathrm{C}_{2} \mathrm{H}_{2}-\mathrm{O}_{2}$ mixture with needle perturbation showing the test matrix and all go/no go data, i.e., successful and unsuccessful transmission of the detonation wave from the confined circular tube to the open area in the spherical chamber. In the present analysis, the critical pressure is defined by the upper limit boundary above which at least $75 \%$ of tests at the same initial condition gives a successful transmission of the detonation wave into the open space. Using all the statistical data obtained in the present investigation, summary of all go/no go results are plotted in Fig. 6 .

Figure 6a) first shows the results for the $\mathrm{C}_{2} \mathrm{H}_{2}-\mathrm{N}_{2} \mathrm{O}$ mixture and the critical pressure limits with and without perturbation for all three tube diameters $D$. For this mixture, it can readily be seen that the perturbation has an influence on the critical tube diameter phenomenon by lowering the critical pressure values for the successful transmission. The reduction in critical pressure caused from the perturbation for the three tubes are 2, 4 and $12 \mathrm{kPa}$, respectively for $D=15.5,12.7$ and $9.13 \mathrm{~mm}$ (or equivalently about $3.0,4.8$ and $9.8 \%$ difference where $\%$ difference is defined by $[100 \%-(x / y \cdot 100 \%)]$ where $x$ and $y$ are the lower and higher number). Similar behaviors are observed for the undiluted stoichiometric $\mathrm{C}_{2} \mathrm{H}_{2}-\mathrm{O}_{2}$ mixture, shown in Fig. 6b). The difference in critical pressure between the perturbed and unperturbed cases are respectively 2,3 and $6 \mathrm{kPa}$ (or equivalently about $15.4,20.0$ and $30.0 \%$ difference) for the three tube diameters $D=15.5,12.7$ and $9.13 \mathrm{~mm}$. From the results of both undiluted irregular mixtures, it appears that the effect of perturbation by the small needle is more apparent for the smallest size tube (i.e., $D=9.13 \mathrm{~mm}$ ) used in this study despite the fact that the blockage ratio is kept the same for all three tube diameter experiments. Although the amount of disturbance was not quantitatively mea30 sured in this study, its trend suggests an agreement for all three scales $D$ and the flow perturbation induced by the needle appears to promote transmission resulting in the decrease in critical pressure. Another observation from the results is that although $\mathrm{C}_{2} \mathrm{H}_{2}-\mathrm{N}_{2} \mathrm{O}$ is more unstable compared to $\mathrm{C}_{2} \mathrm{H}_{2}-\mathrm{O}_{2}$, the effect of the perturbation by the needle seems to be more significant for the latter mixture. Some explanation can perhaps be made within the scope of the spontaneous flame concept, originally proposed by Zel'dovich [24-26]. According to this concept, the origin of explosion center and the mechanism leading to the onset of detonation is conditioned by the gradients of self-ignition time delay in the reactive fluid. Later studies also show that in order for any hydrodynamic fluctuation to grow or eventually initiate a detonation, the disturbance must be sufficiently strong and the critical size must be long on the order of the reaction scale to induce a gradient of thermal ignition time [27-29]. The later condition leads to the coupling between the propagation and amplification of the disturbance with the chemical energy release in the reactive medium [30]. Table 1 shows the relative comparison between the size of the perturbation, roughly estimated by the integral scale of the needle diameter $d_{\text {needle, }}$ to the chemical induction length scale of the two unstable mixtures $\Delta_{I}$ at the critical pressures for the normal case without perturbation. As shown in the table, the ratio $\left(d_{\text {needle }} / \Delta_{I}\right)_{\text {critical }}$ for the $\mathrm{C}_{2} \mathrm{H}_{2}-\mathrm{O}_{2}$ mixture is bigger than the $\mathrm{C}_{2} \mathrm{H}_{2}-\mathrm{N}_{2} \mathrm{O}$ mixture for all three tube diameters $D$. The bigger value of the ratio $\left(d_{\text {needle }} / \Delta_{I}\right)_{\text {critical }}$ for the $\mathrm{C}_{2} \mathrm{H}_{2}-\mathrm{O}_{2}$ mixture therefore suggests that the perturbation is large enough and has turbulent strength, consequently it can reside longer on the order of the chemical induction time and is more effective to promote a spontaneous explosion center that supports the transmission of the detonation into the unconfined area. 
Table 1 Comparison of the ZND induction length with the size of the perturbation at critical conditions. The induction length $\Delta_{I}$ is computed using the San Diego chemical mechanism [22].

\begin{tabular}{cccccc}
\hline Mixture & $D(\mathrm{~mm})$ & $d_{\text {needle }}(\mathrm{mm})$ & $p_{\text {critical }}(\mathrm{kPa})$ & $\Delta_{I}(\mathrm{~mm})$ & $\left(d_{\text {needle }} / \Delta_{I}\right)_{\text {critical }}$ \\
\hline $\mathrm{C}_{2} \mathrm{H}_{2}+5 \mathrm{~N}_{2} \mathrm{O}$ & 15.5 & 1.0 & 67 & $8.74 \times 10^{-2}$ & 11.4 \\
& 12.7 & 0.8 & 85 & $6.77 \times 10^{-2}$ & 11.8 \\
& 9.13 & 0.6 & 124 & $4.62 \times 10^{-2}$ & 13.0 \\
$\mathrm{C}_{2} \mathrm{H}_{2}+2.5 \mathrm{O}_{2}$ & 15.5 & 1.0 & 14 & $5.23 \times 10^{-2}$ & 19.1 \\
& 12.7 & 0.8 & 16 & $4.40 \times 10^{-2}$ & 18.2 \\
& 9.13 & 0.6 & 21 & $3.22 \times 10^{-2}$ & 18.6 \\
\hline
\end{tabular}

On the other hand, the results of Fig. 6c) show that the transmission of detonation from the unconfined tube to the open area in the $\mathrm{C}_{2} \mathrm{H}_{2}-\mathrm{O}_{2}$ mixture highly diluted with argon appears not to be affected by the perturbation. The difference in critical pressure between the perturbed and unperturbed cases in all three tube diameters is less than $1 \mathrm{kPa}$ or approximately $1 \%$. Taking into account the experimental uncertainty which includes the metering panel for the initial filled pressure measurement, it can be concluded that for this argon diluted mixture, essentially the same critical pressure limits between successful and unsuccessful transmission of diverging detonations in the open space are obtained for all tube diameters. Therefore, these results demonstrate that the critical condition for successful detonation transmission is not very sensitive to the flow perturbation by needle.

For the undiluted mixtures where the reaction kinetics is sensitive to flow disturbances and the detonation propagation or transmission relies on the instabilities at the front, i.e., role of the transverse waves, the additional flow perturbations created by the needle compensate the instability suppressed/quenched by the failure wave for the case without perturbation and re-generate local explosion center for successful transmission. In contrast, for diluted mixtures of which the detonation front is very regular or stable and the role of instability does not play a prominent role, the failure mechanism is dominantly caused by the global curvature and as the numerical results in the following qualitatively show, the needle with minimal blockage ratio and the flow disturbance induced does not affect significantly the wave front curvature.

To illustrate qualitatively the effect of the minute needle on the gasdynamic flow field in the experiment, numerical simulation using a simplified two-dimensional geometry was conducted. It is important to emphasize that the numerical simulation is not intended to reproduce the full reactive flow phenomenon of detonation diffraction, which is computationally expensive and often under-resolved. The purpose of the present simple but reliable inert shock simulation is to illustrate qualitatively that the obstacle does not have a global effect of the leading diffracted wave curvature, hence it does not influence the failure mechanism of the diffracted detonation in diluted mixture. Approximating the solutions of
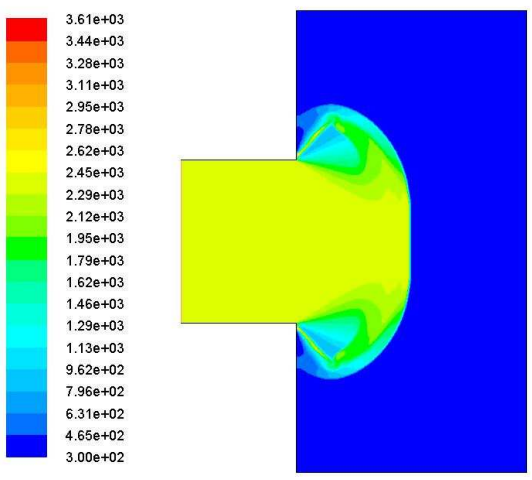

(a)
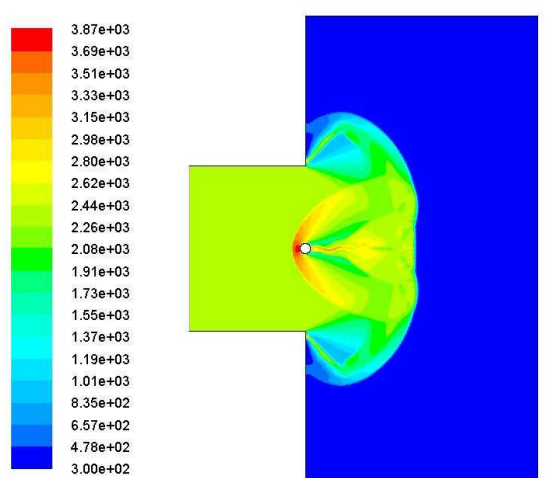

(b)

Fig. 7 Temperature contour plots from the numerical simulation of the diffraction of a Mach 6 shock in air. a) unperturbed case; and b) perturbed case with a small pin obstacle.

Euler equations by the 2nd order Roe's flux difference splitting (FDS) scheme with spatial resolution of 0.06 $\mathrm{mm}$ and CFL number of 0.5 using the software ANSYSFLUENT [31], it was possible to simulate a Mach $M=$ 6 shock diffraction in air $(\gamma=1.4)$ with the interaction of a cylinder at the exit of the channel. Although this represents only a two-dimension simulation, the physical dimensions were kept as close to those used in the experiments. The small channel and the opening chamber have a width of $9 \mathrm{~mm}$ and $25 \mathrm{~mm}$, respectively and the cylindrical obstacle has a diameter of $0.6 \mathrm{~mm}$. To illustrate the gasdynamic change caused by the small obstacle, Fig. 7 shows the temperature contours from the numerical sim- 
Table 2 Numerical values of different parameters and comparison between the drag energy $E_{D}$ with the initiation energy $E_{s}$ at the critical condition for detonation transmission.

\begin{tabular}{cccccccc}
\hline Mixture & $D(\mathrm{~mm})$ & $d_{\text {needle }}(\mathrm{mm})$ & $p_{\text {critical }}(\mathrm{kPa})$ & $p_{C J}(\mathrm{kPa})$ & $\rho_{C J}\left(\mathrm{~kg} / \mathrm{m}^{3}\right)$ & $u_{C J}(\mathrm{~m} / \mathrm{s})$ & $E_{D} / E_{s}(\%)$ \\
\hline $\mathrm{C}_{2} \mathrm{H}_{2}+2.5 \mathrm{O}_{2}+70 \% \mathrm{Ar}$ & 15.5 & 1.0 & 75 & 1,727 & 2.023 & 810 & 3.26 \\
& 12.7 & 0.8 & 89 & 2,062 & 2.399 & 813 & 3.08 \\
& 9.13 & 0.6 & 117 & 2,740 & 3.152 & 817 & 3.21 \\
$\mathrm{C}_{2} \mathrm{H}_{2}+2.5 \mathrm{O}_{2}$ & 15.5 & 1.0 & 14 & 437 & 0.317 & 1,065 & 3.38 \\
& 12.7 & 0.8 & 16 & 502 & 0.362 & 1,068 & 3.30 \\
$\mathrm{C}_{2} \mathrm{H}_{2}+5 \mathrm{~N}_{2} \mathrm{O}$ & 9.13 & 0.6 & 21 & 666 & 0.475 & 1,074 & 3.44 \\
& 15.5 & 1.0 & 67 & 2,587 & 2.038 & 1,018 & 3.35 \\
& 12.7 & 0.8 & 85 & 3,310 & 2.585 & 1,022 & 3.27 \\
\hline
\end{tabular}

ulation. Without the pin obstacle, the wave diffracts and the expansion waves continues to enter the center core and reduces the flow temperature behind the wave front, see Fig. 7a). For perturbed case shown in Fig. 7b), upon the wave interaction with the small obstacle, a number of transverse waves disturbances are generated and regions (including the front) with localized temperature fluctuation can be seen. However, the global curvature of the diffracted wave does not change much. As explained earlier for unstable mixtures with high reaction sensitivity, these hydrodynamic fluctuations will have an effect on the detonation diffraction to promote local explosion centers for successful transmission. In contrast to regular mixtures where instabilities are not the dominant mechanism in transmission, once the leading front fails due to the excessive curvature from the diffraction, the perturbation will not be able to give rise to a transmitted detonation.

Theoretically, it may perhaps be possible to explain the observation using an energy-drag approach, an analysis similar to detonation initiation by high speed projectile [32-34]. Through the drag force, the small obstacle can deposit an amount of energy into the product flow mixture. The drag force of the needle of diameter $d_{\text {needle }}$ and length $l$ (which is equal to the diameter of the detonation tube $D$ ) can be written as:

$F_{D}=\frac{1}{2} C_{D} A_{f} \rho_{C J} u_{C J}^{2}$

where $F_{D}$ is the drag force, $A_{f}$ is the frontal or projected area (here for a cylinder of length $L=D$, this area is equal to $\left.d_{\text {needle }} \cdot D\right), C_{D}$ the drag coefficient and $\rho_{C J}, u_{C J}$ are the CJ velocity and particle velocity of the detonation product flow, respectively. Because the freestream cross-flow is of high Reynolds number, the drag coefficient of the cylindrical needle takes approximately on a number value of 1.0. Lee's work done model [35] can then be used to estimate the energy deposition given by:

$E_{D}=\int_{0}^{t^{*}} F_{D} u_{C J} d t$ where $t^{*}$ is modeled as the time when the rarefaction wave reaches the tube axis, which can be approximately by $t^{*} \sim D / 2 a_{C J}$ with $a_{C J}$ being the sound speed of the detonation products [35-36]. Hence, using the drag force,

$E_{D}=\frac{C_{D} \rho_{C J} u_{C J}^{3} d_{\text {needle }} D^{2}}{4 a_{C J}}$

It is of interest to compare this work done by drag to the critical energy required to initiate a spherical detonation in the unconfined space. Based on the work done model by Lee [37-39], it is assumed that the energy needed to reinitiate a detonation downstream of the unconfined space in the critical tube diameter problem can be related to the work done delivered by the detonation product in the confined tube (i.e., a fictitious piston) over the same period $t^{*}$ given above, the energy can be obtained by:

$E_{s}=\int_{0}^{t^{*}} p_{C J} \frac{\pi D^{2}}{4} u_{C J} d t$

where $p_{C J}$ and $u_{C J}$ denote the CJ detonation pressure and particle velocity, respectively. After the integration the simplified work done model thus gives:

$E_{s}=\frac{p_{C J} u_{C J} \pi D^{3}}{8 a_{C J}}$

By comparing both energies $E_{D}$ and $E_{s}$ the following expression is obtained:

$\frac{E_{D}}{E_{s}}=\frac{2 C_{D} \rho_{C J} u_{C J}^{2} d_{\text {needle }}}{\pi p_{C J} D}$

Based on this expression, Table 2 shows values of different parameters and compares the drag energy with the initiation energy at the critical condition for successful transmission in the unconfined area. The present estimation shows that the drag (energy) is at most $\sim 3 \%$ (found to be roughly same for both diluted and undiluted mixtures) of that the mean detonation product flow responsible for the initiation in the free space. This $3 \%$ is indeed negligible compared to the initiation energy change required for the observed decrease in critical pressures in 
undiluted mixtures. Such decrease in near critical pressure is equivalent to far more than $50 \%$ increase in initiation energy for a diverging detonation [40]. Therefore, a global energy-drag approach is not a possible interpretation of physics for the present results. Although with the tiny obstacle some kinetic energy got converted to thermal one persisting downstream - resulted in local fluctuation or instability that can promote the transition in the undiluted mixtures but play no effect for lesstemperature sensitive diluted mixtures with the failure due to the global curvature of the diverging wave only. These mechanisms are confirmed in this study by experiments and such confirmation represents the significant merit of this study.

Experiments were also carried out with other perturbation geometries, i.e., different configurations of the needle obstacle while keeping the overall blockage ratio constant. It can be seen from Figs. 8 and 9 showing the results for two different tube diameters $D=15.5$ and $9.13 \mathrm{~mm}$ that the perturbation effect is geometry independence of the obstacle. In other words, as the blockage ratio for a specific tube is kept constant, regardless of its geometry or needles configuration, results show almost identical behavior. For the irregular mixtures all the results with different needle(s) perturbations show similar decrease in critical pressure for successful transmission. On the other hand, for the mixture highly diluted with argon, where it has been suggested that the cellular instabilities play minor roles in the detonation propagation mechanism, the flow perturbation (despite different arrangement) does not have any major effect on the phenomenon.

\section{Concluding remarks}

In the present investigation, the critical tube diameter phenomenon and the failure mechanism for detonation diffraction in three combustible mixtures, ranging from highly irregular to regular detonation structures are studied. Gasdynamic disturbances were introduced using a needle with small blockage ratio at the exit of the tube before the gaseous detonation emerges into the free unconfined space. By observing how the detonation responds to the flow perturbation during the diffraction, it is possible to investigate the important role of instability and to provide confirmation on the two postulated modes of failure mechanism proposed by Lee [14] on the phenomena of critical tube diameter. For the cases of undiluted stoichiometric $\mathrm{C}_{2} \mathrm{H}_{2}-\mathrm{N}_{2} \mathrm{O}$ and $\mathrm{C}_{2} \mathrm{H}_{2}-\mathrm{O}_{2}$ mixtures in which the detonation wave is highly unstable and relies on the instability at the cellular front, it is found that the additional flow perturbation can cause a noticeable effect on the detonation diffraction. Despite a difference due to the scale effect, all the results of the three tube diameters with needle perturbations show a decrease in critical pressure for successful transmission. On the other

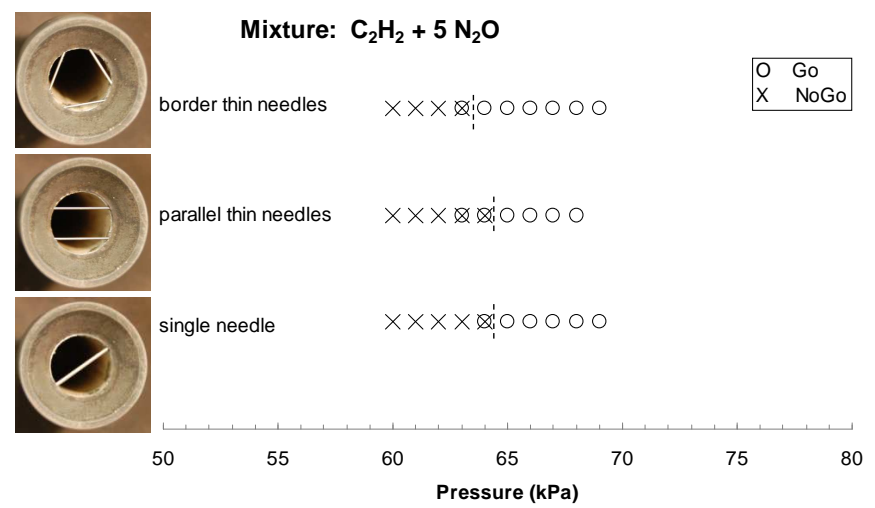

(a)

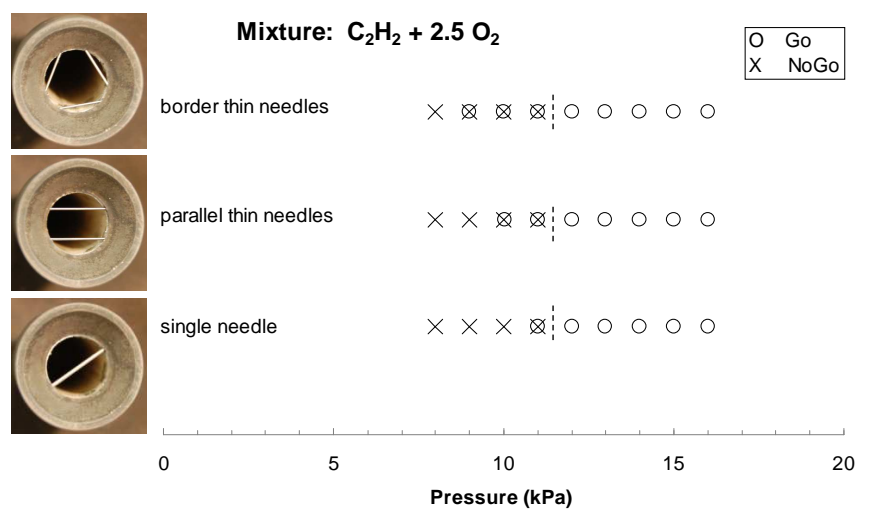

(b)

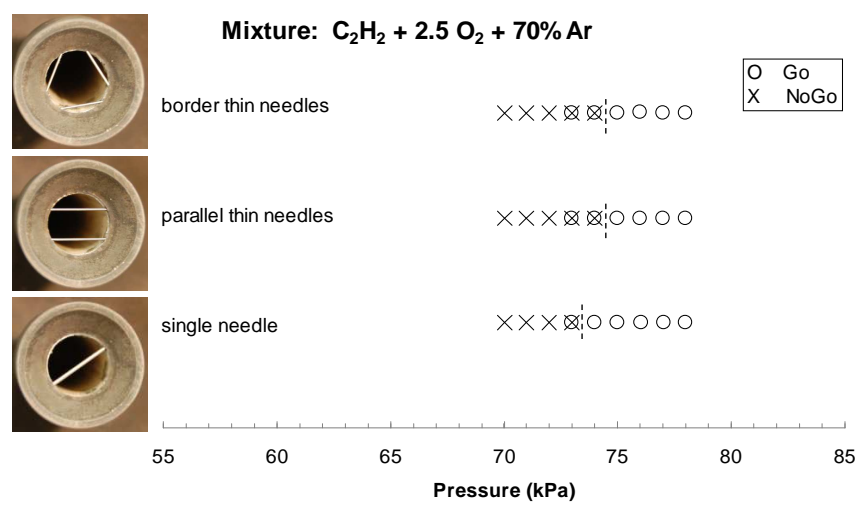

(c)

Fig. 8 Summary of go/no go results for all three combustible mixtures with different needle arrangements $(B R \sim 0.08)$ to create perturbation and tube diameter $D=15.5 \mathrm{~mm}$.

hand, for the mixture highly diluted with argon - where it has been suggested that the cellular instabilities play minor roles in the detonation propagation mechanism, the flow perturbation does not have any major effect on the phenomenon. Numerical simulations show that the hydrodynamic disturbance induced by the needle provides flow fluctuation behind the wave but does not significantly change the curvature of the diffracted wave. 


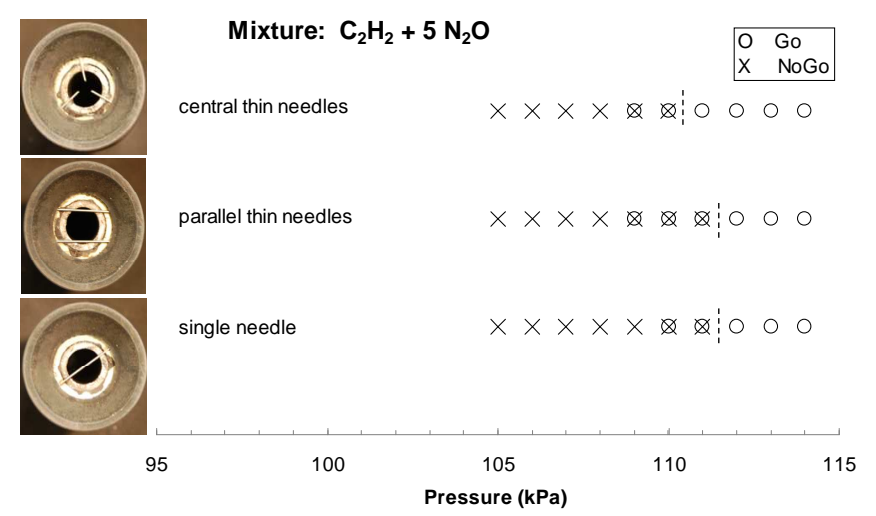

(a)

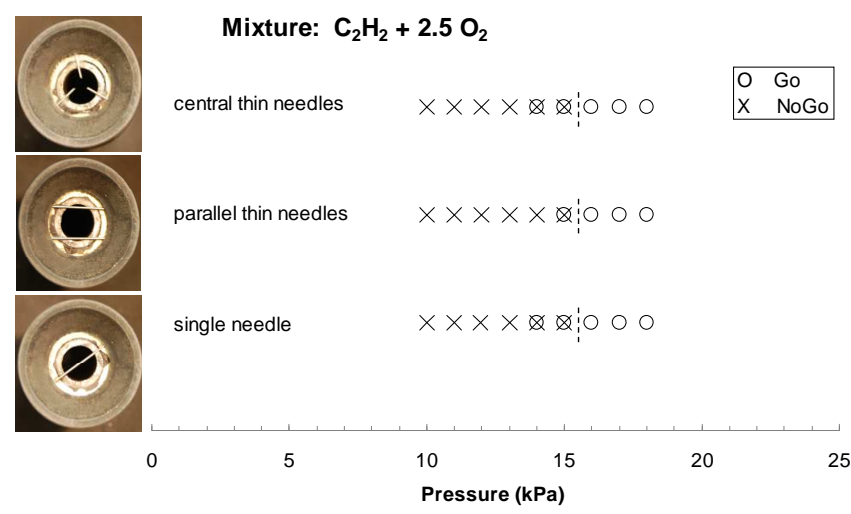

(b)

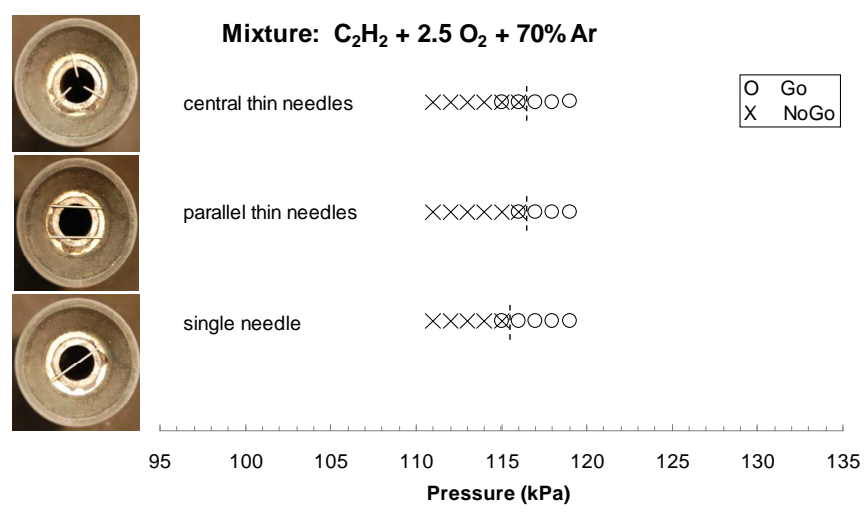

(c)

Fig. 9 Summary of go/no go results for all three combustible mixtures with different needle arrangements $(\mathrm{BR} \sim 0.08)$ to create perturbation and tube diameter $D=9.13 \mathrm{~mm}$.

This result appears to support a curvature based global mechanism for diffraction failure in these stable mixtures rather than the suppression of instability by the failure wave and the inability to generate local explosion centers. Finally, the present results also show that while the blockage ratio for a specific tube is kept constant and relatively small, essentially the same experimental observation is obtained for different blockage configura- tions (i.e., needle arrangement), which do not affect the conclusion of this study.

Acknowledgements This work is supported by the Fonds de Recherche du Québec - Nature et Technologies.

\section{References}

1. Lee, J.H.S.: Dynamic parameters of gaseous detonations. Annu. Rev. Fluid Mech. 16, 311-336 (1984).

2. Vasil'ev, A.A.: Dynamic parameters of detonation. In: F. Zhang, Editor, Shock Waves Science and Technology Library, Vol 6: Detonation Dynamics, Springer, Berlin Heidelberg (2012) Chap. 4.

3. Roy G.D., Frolov, S.M., Borisov, A.A., Netzer, D.W.: Pulse detonation propulsion: challenges, current status, and future perspective. Prog. Energy Combust. Sci. 30, 545-672 (2004)

4. Mitrofanov, V.V., Soloukhin, R.I.: The diffraction of multifront detonation waves. Sov. Phys.-Dokl. 9(12), 1055 (1965).

5. Knystautas, R., Lee, J.H., Guirao, C.: The critical tube diameter for detonation failure in hydrocarbon-air mixtures. Combust. Flame 48, 63-83 (1982).

6. Desbordes, D., Guerraud, C., Hamada, L., Presles, H.N.: Failure of classical dynamic parameters relationships in highly regular cellular detonation systems. Prog. Astronaut. Aeronaut. 153, 347-359 (1993).

7. Moen, I., Sulmistras, A., Thomas, G.O., Bjerketvedt, D., Thibault, P.A.: Influence of cellular regularity on the behavior of gaseous detonations. Prog. Astronaut. Aeronaut. 106, 220-243 (1986).

8. Shepherd, J.E., Moen, I., Murray, S., Thibault, P.I.: Analyses of the cellular structure of detonations. Proc. Combust. Inst. 21, 1649-1658 (1986).

9. Shepherd, J.E.: Detonation in gases. Proc. Proc. Combust. Inst. 32, 83-98 (2009).

10. Pintgen, F., Eckett, C.A., Austin, J.M., Shepherd, J.E.: Direct observations of reaction zone structure in propagating detonations, Combust. Flame 133(3), 211-229 (2003).

11. Radulescu, M.I.: The propagation and failure mechanism of gaseous detonations: experiments in porous-walled tubes. Ph.D. thesis, McGill University, Canada (2003).

12. Radulescu, M.I., Ng, H.D., Varatharajan, B., Lee, J.H.S.: The effect of argon dilution on the stability of acetyleneoxygen detonations. Proc. Combust. Inst. 29, 2825-2831 (2002).

13. Ng, H.D., Radulescu, M.I., Higgins, A.J., Nikiforakis, N., Lee, J.H.S.: Numerical investigation of the instability for one-dimensional Chapman-Jouguet detonations with chain-branching kinetics. Combust. Theory Model. 9, 385401 (2005).

14. Lee, J.H.S.: On the critical tube diameter. In: Bowen, J. (ed.) Dynamics of Exothermicity, pp. 321 Gordon and Breach, Amsterdam (1996).

15. Lee, J.H.S.: Detonation Waves in Gaseous Explosives. In: G. Ben-Dor, O. Igra, T. Elperin, Editors, Handbook of Shock Waves, Volume 3, Chapter 17, 309-415 (2001).

16. Kamenskihs, V., Ng, H.D., Lee, J.H.S.:Measurement of critical energy for direct initiation of spherical detonations in high-pressure H2-O2 mixtures. Combust. Flame, 157(9), 1795-1799 (2010).

17. Zhang, B., Kamenskihs, V., Ng, H.D., Lee, J.H.S.: Direct blast initiation of spherical gaseous detonation in highly argon diluted mixtures. Proc. Combust. Inst., 33(2), 22652271 (2011). 
18. Zhang, B., Ng, H.D., Mével, R., Lee, J.H.S.: Critical energy for direct initiation of spherical detonations in $\mathrm{H} 2 / \mathrm{N} 2 \mathrm{O} / \mathrm{Ar}$ mixtures. Int. J. Hydrogen Energy 36, 57075716 (2011).

19. Zhang, B., Ng, H.D., Lee, J.H.S.: Direct measurement and relationship between critical tube diameter and critical energy for direct detonation initiation. 23rd International Colloquium on the Dynamics of Explosions and Reactive Systems, Irvine, US, July 24-July 29, (2011).

20. Ng, H.D., Zhang, F.: Detonation Instability. In: F. Zhang, Editor, Shock Waves Science and Technology Library, Vol 6: Detonation Dynamics, Springer, Berlin Heidelberg (2012) Chap. 3.

21. Kee, R.J., Rupley, F.M., Miller, J.A.: A Fortran chemical kinetics package for the analysis of gas-phase chemical kinetics. Sandia National Laboratories report SAND89-8009 (1989).

22. San Diego Mechanism web page, Mechanical and Aerospace Engineering (Combustion Research), University of California at San Diego (http://combustion.ucsd.edu).

23. Varatharajan, B., Williams, F.A.: Chemical-kinetic descriptions of high-temperature ignition and detonation of acetylene-oxygen diluent systems. Combust. Flame 124(4), 624-645 (2001).

24. Zel'dovich, Ya. B., Librovich, V. B., Makhviladze, G.M., Sivashinsky, G.I.: Development Of detonation In a nonuniformly preheated gas. Astron. Acta 15, 313-321 (1970).

25. Zel'dovich, Ya. B.: Regime classification of an exothermic reaction with non- uniform initial conditions. Combust. Flame, 39, 211-214 (1980).

26. Bartenev, A.M., Gelfand, B.E.: Spontaneous initiation of detonations. Prog. Energy Combust. Sci. 26(1), 29-55 (2000).

27. Short, M.: On the critical conditions for the initiation of a detonation in a nonuniformly perturbed reactive fluid. SIAM J. Appl. Math. 57(5), 1242-1280 (1997)

28. Khokhlov, A.M., Oran, E.S., Wheeler, J.C.: A theory of DDT in unconfined flames. Combust Flame 108, 503-517 (1997).

29. Kapila, A.K., Schwendeman, D.W., Quirk, J.J., Hawa, T.: Mechanisms of detonation formation due to a temperature gradient. Combust. Theory Model. 6, 553-594 (2002).

30. Lee J.H.S., Knystautas R., Yoshikawa N.: Photochemical initiation of gaseous detonations. Acta Astro. 5, 971-982 (1978).

31. ANSYS Inc. Fluent 12.0 Canonsburg, PA. 12.0 Users' Guide, 2009.

32. Vasiljev, A.A.: Initiation of gaseous detonation by a high speed body. Shock waves 3, 321-326 (1994).

33. Lee, J.H.S.: Initiation of detonation by a hypervelocity projectile. Prog. Astronaut. Aeronaut. 173, 293-310 (1997).

34. Verreault, J., Higgins, A.J.: Initiation of Detonation by Conical Projectiles. Proc Combust. Inst. 33(2), 2311-2318 (2011).

35. Matsui, H., Lee, J.H.S.: On the measure of the relative detonation hazards of gaseous fuel-oxygen and air mixtures. Proc. Combust. Inst. 17, 1269-1280 (1978).

36. Vasil'ev, A.A.: Diffraction estimate of the critical energy for initiation of gaseous detonation. Combust. Expl. Shock Waves 34(4), 433-437 (1998).

37. Desbordes, D.: Transmission of overdriven plane detonations: critical diameter as a function of cell regularity and size. Prog. Astronaut. Aeronaut. 11, 170-185 (1988).

38. Sochet, I., Lamy, T., Brossard, J., Vaglio, C., Cayzac, R.: Critical tube diameter for detonation transmission and critical initiation energy of spherical detonation. Shock Waves 9, 113-123 (1999).
39. Zhang, B., Ng, H.D., Lee, J.H.S.: Measurement of effective blast energy for direct initiation of spherical gaseous detonations from high-voltage spark discharge. Shock Waves 22(1) 1-7 (2012).

40. Zhang, B., Ng, H.D., Lee, J.H.S.: Measurement and scaling analysis of critical energy for direct initiation of detonation. Shock Waves 22(3), 275-279 (2012). 